\title{
Principais diagnósticos de enfermagem em pacientes com manifestações clínicas da COVID-19
}

\author{
Key nursing diagnoses in patients with clinical manifestations of COVID-19 \\ Diagnósticos principales de enfermería en pacientes con manifestaciones clínicas de \\ COVID-19
}

Thandara Rejane Santos Ferreira Andrade ${ }^{1 *}$, lanka Heloisa Alencar Santos ${ }^{1}$, Grayce Ellen Souza Rezende $^{1}$, Erivelton Cunha Torres ${ }^{1}$, Cleidinaldo Ribeiro de Goes Marques ${ }^{2}$, Edna Santos Dias ${ }^{1}$, Aline Barreto Hora ${ }^{1}$, Anderson Batista Cavalcante ${ }^{3}$, Carla Viviane Freitas de Jesus ${ }^{1}$, Yasmim Anayr Costa Ferrari².

\section{RESUMO}

Objetivo: Relacionar os aspectos clínicos e a sintomatologia dos pacientes com os diagnósticos de enfermagem aplicados no contexto da COVID-19 através da taxonomia da Associação Norte-Americana de Diagnósticos de Enfermagem Internacional (NANDA-I). Métodos: Pesquisa exploratória e bibliográfica. A partir das manifestações clínicas descritas na literatura como as mais utilizadas para triagem da COVID-19, foram pesquisados e elaborados, por enfermeiros capacitados e com prática clínica junto ao paciente com COVID-19, os principais diagnósticos de enfermagem para os pacientes acometidos pela doença a partir da taxonomia da NANDA-I. Resultados: As manifestações clínicas escolhidas foram tosse, febre, mialgia, dispneia, dor de garganta, fadiga e coriza. Foram selecionados 13 diagnósticos de enfermagem pertencentes a quatro domínios: domínio 2 - nutrição, domínio 4 - atividade/repouso, domínio 11 - segurança/proteção e domínio 12 - conforto. Considerações Finais: A Sistematização da Assistência de Enfermagem e o Processo de Enfermagem se destacam por permitir o cuidado holístico ao paciente com COVID-19, com acompanhamento diário e estruturado. Os diagnósticos de enfermagem elencados neste estudo para as manifestações clínicas da doença englobaram fatores nutricionais, respiratórios, de conforto e bem-estar, todos esses elementos importantes para a recuperação do indivíduo infectado pelo coronavírus.

Palavras-chave: Infecções por coronavírus, Diagnóstico de enfermagem, Enfermeiras e enfermeiros, Coronavírus.

\section{ABSTRACT}

Objective: To relate the clinical aspects and the symptomatology of patients with the nursing diagnoses applied in the context of COVID-19 through the taxonomy of the North American Association of International Nursing Diagnoses (NANDA-I). Methods: Exploratory and bibliographic research. From the clinical manifestations described in the literature as the most used for screening of COVID-19, trained nurses with clinical practice with the patient with COVID-19 were researched and developed, the main nursing diagnoses for patients affected by the disease from the NANDA-I taxonomy. Results: The clinical manifestations chosen were cough, fever, myalgia, dyspnea, sore throat, fatigue and runny nose. 13 nursing diagnoses were selected, belonging to four domains: domain 2 - nutrition, domain 4 - activity/rest, domain 11 - safety/protection and domain 12 - comfort. Final Considerations: The Systematization of Nursing Assistance and the Nursing Process stand out for allowing holistic care to patients with COVID-19, with daily and structured monitoring. The nursing diagnoses listed in this study for the clinical manifestations of the disease included nutritional, respiratory, comfort and well-being factors, all of these important elements for the recovery of the individual infected with the coronavirus.

Keywords: Coronavirus infections, Nursing diagnosis, Nurses, Coronavirus.

\footnotetext{
${ }^{1}$ Universidade Tiradentes (UNIT), Aracaju - SE. *E-mail: thandara456@outlook.com

2Universidade Federal de Sergipe (UFS), Aracaju - SE.

${ }^{3}$ Centro Universitário Estácio de Sergipe, Aracaju - SE.
} 


\section{RESUMEN}

Objetivo: Relacionar los aspectos clínicos y la sintomatología de los pacientes con los diagnósticos de enfermería aplicados en el contexto de COVID-19 a través de la taxonomía de la Asociación Norteamericana de Diagnósticos Internacionales de Enfermería (NANDA-I). Métodos: Investigación exploratoria y bibliográfica. A partir de las manifestaciones clínicas descritas en la literatura como las más utilizadas para la detección de COVID-19, se investigaron y prepararon enfermeras capacitadas con práctica clínica con el paciente con COVID-19, los principales diagnósticos de enfermería para pacientes afectados por la enfermedad de la taxonomía NANDA-I. Resultados: Las manifestaciones clínicas elegidas fueron tos, fiebre, mialgia, disnea, dolor de garganta, fatiga y secreción nasal. Se seleccionaron 13 diagnósticos de enfermería, pertenecientes a cuatro dominios: dominio 2 - nutrición, dominio 4 - actividad/descanso, dominio 11 seguridad/protección y dominio 12 - comodidad. Consideraciones Finales: La Sistematización de la Atención de Enfermería y el Proceso de Enfermería se destacan por permitir una atención integral a los pacientes con COVID-19, con un seguimiento diario y estructurado. Los diagnósticos de enfermería enumerados en este estudio para las manifestaciones clínicas de la enfermedad abarcaron factores nutricionales, respiratorios, de confort y bienestar, todos estos elementos importantes para la recuperación del individuo infectado con el coronavirus.

Palabras clave: Infecciones por coronavirus, Diagnóstico de enfermería, Enfermeras y enfermeros, Coronavirus.

\section{INTRODUÇÃO}

Os coronavírus fazem parte de um relevante grupo de vírus, evidenciados e isolados no ano de 1937 . Em 1965 o vírus foi descrito em decorrência do aspecto microscópico semelhante a uma coroa, por conseguinte recebeu a nomenclatura corona proveniente do latim "coroa". Segundo a Organização Mundial da Saúde (OMS), em 2002, o SARS-CoV foi transmitido por gatos selvagens para seres humanos na China, por isso o novo agente dos coronavírus que assola a humanidade recebeu o nome SARS-CoV-2, pois assemelha-se geneticamente com o vírus da Síndrome Respiratória Aguda Grave (SARS) de 2002 (DANTAS TP, et al., 2020; FIOCRUZ, 2020; OPAS, 2020).

A pandemia do coronavírus 2 associada à SARS-CoV-2, responsável pelo surgimento da doença do coronavírus 2019 (COVID-19) na cidade de Wuhan, China, no final de 2019, ligeiramente disseminou-se por todos os continentes, aumentando exponencialmente o número de infectados e provocando milhares de mortes no globo. Tendo em vista o agente etiológico da COVID-19, o mesmo apresenta elevada transmissibilidade, disseminando-se através do contato com gotículas e aerossolização liberada em procedimentos invasivos, como intubação orotraqueal, aspiração de vias aéreas e reanimação cardiopulmonar (BITENCOURT GR, et al., 2020).

A sintomatologia da COVID-19 pode variar de uma gripe comum a uma pneumonia, na maioria dos indivíduos os sintomas predominantes são leves. Entretanto, existem vários subtipos do vírus que ocasionam a propensão de maiores riscos de evolução para as formas graves da enfermidade. A OMS afirma que pessoas maiores de 60 anos e com doenças preexistentes possuem maiores riscos de adquirir formas severas do agravo. Algumas das manifestações clínicas que podem ser apresentadas pelos pacientes infectados pelo coronavírus são febre, mialgia, tosse, cefaleia, coriza, dor de garganta, diarreia, fadiga, perda do paladar e do olfato e dispneia. No tocante às manifestações graves, o paciente pode desenvolver complicações pulmonares, tais como a Síndrome da Angústia Respiratória Aguda (SARA) (BRASIL, 2020a; FIOCRUZ, 2020; MONTE LM, et al., 2020; OPAS, 2020).

O diagnóstico da infecção pelo coronavírus é dividido em casos suspeitos e casos confirmados. Os casos suspeitos podem ser definidos como síndrome gripal, onde o indivíduo apresenta sensação febril ou febre que pode ou não ser acompanhada de tosse, coriza, dor de garganta e/ou dificuldade respiratória, e síndrome respiratória aguda grave, quando o paciente apresenta dispneia, saturação de oxigênio inferior a 95\%, pressão permanente no tórax e/ou cianose nos lábios ou rosto. Os casos podem ser confirmados através de critérios laboratoriais com teste de biologia molecular ou imunológico e por critérios clínicos-epidemiológicos, caracterizados como os casos que tiveram contato no domicílio ou local próximo com pessoa diagnosticada laboratorialmente para COVID-19, nos últimos sete dias antes do surgimento das manifestações clínicas, mas 
que não foram testadas com exames laboratoriais para a doença. Os casos descartados são aqueles que tiveram um resultado negativo para o critério laboratorial (BRASIL, 2020a).

Diante do exposto, é importante elencar que a Enfermagem corresponde a maior categoria de profissionais da saúde, além de estar ao lado do paciente em todo processo de cuidado e atendimento prestado. Sendo assim, as ações e medidas tomadas pelo profissional enfermeiro são de destaque para uma assistência clínica e especializada aos pacientes, visto que tal profissional exerce função de coordenação e liderança dos serviços de saúde, guiando a equipe para uma adequada prestação de cuidados de enfermagem, a fim de promover melhores repercussões clínicas aos pacientes (TANNURE MC e PINHEIRO AM, 2010).

Dessa forma, a enfermagem contribui na assistência ao paciente por meio da Sistematização da Assistência de Enfermagem (SAE), que se caracteriza como um método de organização e sistematização do cuidado embasado em princípios científicos, realizada através do pensamento crítico e julgamento clínico. Identificam-se as necessidades e intervenções de enfermagem relacionadas a promoção, prevenção e recuperação do indivíduo. A implementação da SAE proporciona cuidados individualizados e holísticos, além de propiciar avanços e desenvolvimento na qualidade da assistência de enfermagem (TANNURE MC e PINHEIRO AM, 2010; DANTAS TP, et al., 2020).

Como parte da SAE destaca-se o Processo de Enfermagem (PE) que é constituído pela documentação das seguintes etapas: histórico do paciente, diagnóstico de enfermagem, planejamento, implementação e avaliação. Destaca-se a segunda etapa, pois, traçar diagnósticos de enfermagem no contexto da COVID-19 é de suma importância para um cuidado integral quanto as necessidades dos indivíduos.

Os diagnósticos de enfermagem possuem diversas linguagens taxonômicas, entre elas a da Associação Norte-Americana de Diagnósticos de Enfermagem Internacional (NANDA-I), que se constitui como uma das principais, sendo padronizada e atualizada de acordo com as necessidades e a clínica do paciente (BARROS ALBL, et al., 2015; NANDA-I, 2018; BITENCOURT GR, et al., 2020).

Por conseguinte, reflete-se a necessidade de elencar diagnósticos de enfermagem para os pacientes acometidos pela COVID-19, a fim de sistematizar e priorizar os cuidados de acordo com as manifestações clínicas apresentadas e visualizar o paciente de maneira holística. Para tal, o objetivo do presente estudo é relacionar os aspectos clínicos e a sintomatologia dos pacientes com os diagnósticos de enfermagem aplicados no contexto da COVID-19 através da taxonomia da Associação Norte-Americana de Diagnósticos de Enfermagem Internacional (NANDA-I) versão 2018-2020.

\section{MÉTODOS}

Trata-se de uma pesquisa exploratória e bibliográfica. A partir das manifestações clínicas descritas na literatura como as mais utilizadas para triagem da COVID-19, foram pesquisados e elaborados os principais diagnósticos de enfermagem possíveis para os pacientes acometidos pela doença a partir da taxonomia da NANDA-I (NANDA-I, 2018). A taxonomia da NANDA-I é composta por 13 domínios, 47 classes e 244 diagnósticos de enfermagem. Os domínios são referentes aos temas centrais, uma área de interesse sobre determinado tema; as classes são subtemas dos domínios; e, os diagnósticos de enfermagem representam a resposta clínica do indivíduo/família/grupo/comunidade à determinada condição de saúde e/ou situação da vida (NANDA-I, 2018).

Os diagnósticos de enfermagem podem ser classificados em: diagnóstico com foco no problema, diagnóstico de risco, diagnóstico de promoção da saúde e diagnóstico de síndrome. A composição é feita através do título do diagnóstico de enfermagem, características definidoras (sinais e sintomas), fatores relacionados (etiologia) ou fatores de risco (determinante de risco), população em risco (grupo de pessoas com risco específico não modificável) e condições associadas (condições que podem influenciar no diagnóstico de enfermagem e não são modificáveis pelo enfermeiro de forma independente), sendo os dois últimos usados somente em diagnósticos que possuem tais fatores (NANDA-I, 2018). O uso dos elementos citados vai variar de acordo com o tipo de diagnóstico, conforme demonstrado na Figura 1. 
Figura 1 - Estrutura dos diagnósticos de enfermagem de acordo com a taxonomia da NANDA-I. Aracaju-SE, 2020.

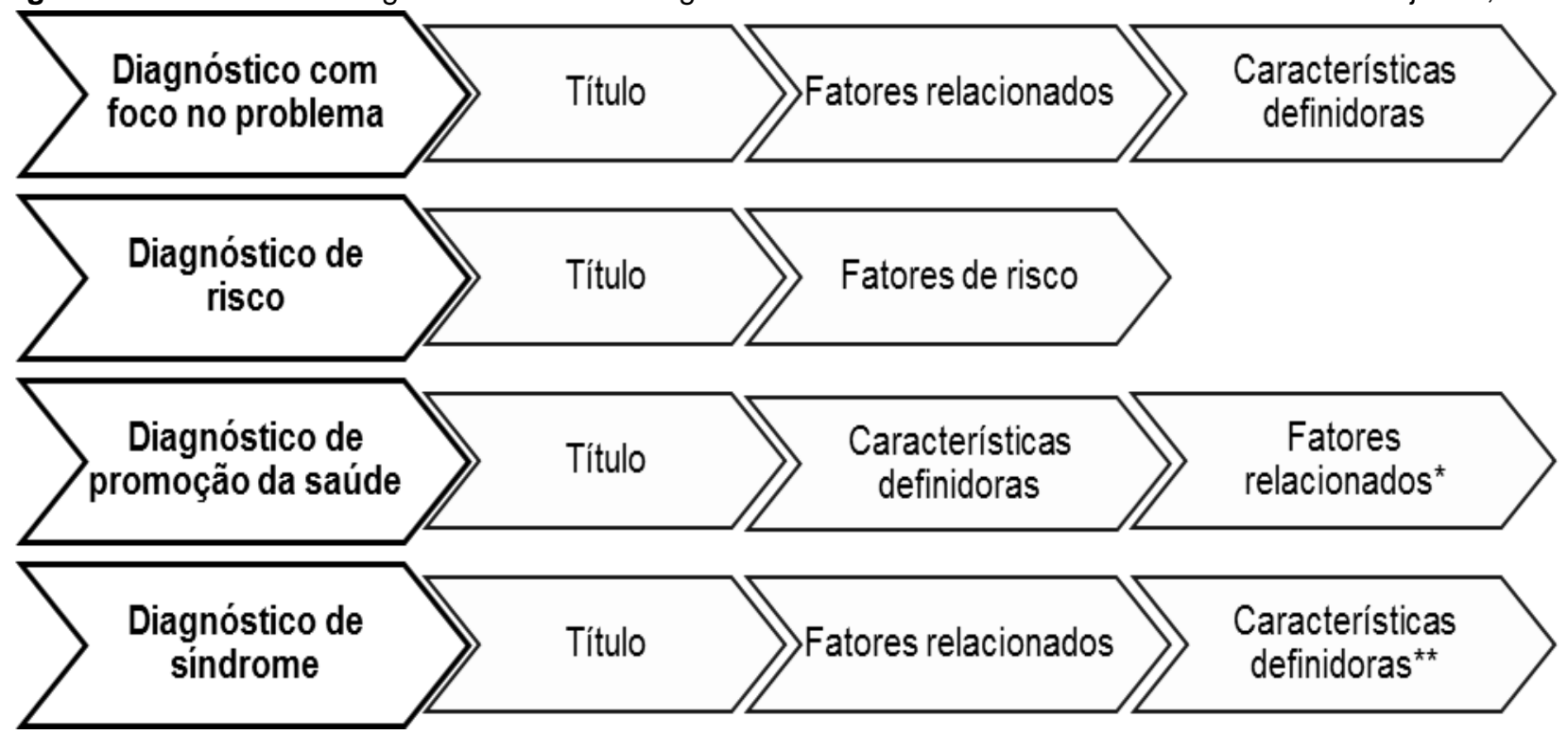

Legenda: * O diagnóstico de promoção da saúde só necessita de fator relacionado se este for útil para esclarecer o diagnóstico. ** Dois ou mais diagnósticos de enfermagem devem ser usados como características definidoras.

Fonte: Andrade TRSF, et al., 2020; dados extraídos de NANDA-I (2018).

A leitura dos diagnósticos de enfermagem é realizada através de termos específicos que sinalizam cada componente do diagnóstico. Para os fatores relacionados/fatores de risco deve ser utilizada a expressão "relacionado a/ao" e para as características definidoras "evidenciado por". O resultado dessa junção de termos fica: título do diagnóstico + relacionado a/ao (fatores relacionados/fatores de risco) + evidenciado por (características definidoras) (NANDA-I, 2018).

A escolha das manifestações clínicas abordadas no presente estudo se deu através da análise das "Diretrizes para diagnóstico e tratamento da COVID-19", elaboradas pelo Ministério da Saúde, a partir dos fluxogramas disponíveis para manejo dos casos suspeitos da COVID-19, onde estão dispostas as manifestações mais comuns da doença, sendo escolhidas a tosse, febre, mialgia, dispneia, dor de garganta, fadiga e coriza (BRASIL, 2020a).

Para melhor abordagem dos termos, a partir do "Manual de semiologia médica: a prática do exame físico" elaborado por Yoshikawa G e Castro RC (2015), as definições para os sinais e sintomas escolhidos foram descritos abaixo: Tosse: mecanismo fisiológico que permite a expectoração de agentes do sistema pulmonar, pode ser classificada como aguda, subaguda e crônica; Febre: temperatura corporal acima dos valores de normalidade, com classificação de febre leve até $37,5^{\circ} \mathrm{C}$, moderada de $37,5-38,5^{\circ} \mathrm{C}$ e alta acima de $38,5^{\circ} \mathrm{C}$; Mialgia: caracterizada como dores nos músculos que podem atingir qualquer parte do corpo; Dispneia: representada pela dificuldade que o indivíduo apresenta para respirar que pode ocorrer desde a realização de pequenos a grandes esforços ou até mesmo nos momentos de repouso; Dor de garganta: envolve a dor (odinofagia) e a dificuldade na deglutição (disfagia); Fadiga: ocorre quando a pessoa tem a sensação de energia diminuída para realizar suas atividades, até mesmo ao fazer pequenos esforços ou em repouso; Coriza: caracterizada pelo corrimento nasal (rinorreia).

Dessa forma, a partir da fisiopatologia da COVID-19 e das manifestações clínicas descritas na literatura como as mais presentes nos pacientes com a doença, enfermeiros capacitados e com prática clínica junto ao paciente com COVID-19 fizeram a análise da taxonomia da NANDA-I para a elaboração dos diagnósticos de enfermagem. Após a avaliação na íntegra da taxonomia da NANDA-I, os pesquisadores elencaram os diagnósticos de enfermagem que mais se enquadravam no objetivo proposto para o estudo. 


\section{RESULTADOS}

Após a delimitação dos sinais e sintomas e análise detalhada da taxonomia NANDA-I foram selecionados 13 diagnósticos de enfermagem pertencentes a quatro domínios, são eles: domínio 2 - nutrição, domínio 4 atividade/repouso, domínio 11 - segurança/proteção e domínio 12 - conforto. O quadro abaixo destaca o domínio ao qual pertence o diagnóstico de enfermagem, o título do diagnóstico de enfermagem, o código do diagnóstico entre parênteses e a sua definição de acordo com a taxonomia NANDA-I (Tabela 1).

Tabela 1 - Domínios, diagnósticos de enfermagem e definições de acordo com manifestações clínicas da COVID-19. Aracaju-SE, 2020.

\begin{tabular}{|c|c|c|}
\hline Domínios & $\begin{array}{l}\text { Diagnósticos de } \\
\text { enfermagem }\end{array}$ & Definições \\
\hline 2 - Nutrição & $\begin{array}{l}\text { Deglutição prejudicada } \\
(00103)\end{array}$ & $\begin{array}{c}\text { Funcionamento anormal do mecanismo da deglutição } \\
\text { associado a déficits na estrutura ou função oral, faríngea ou } \\
\text { esofágica. }\end{array}$ \\
\hline 2 - Nutrição & $\begin{array}{c}\text { Nutrição } \\
\text { desequilibrada: menor } \\
\text { do que as } \\
\text { necessidades } \\
\text { corporais (00002) }\end{array}$ & $\begin{array}{l}\text { Ingestão de nutrientes insuficiente para satisfazer às } \\
\text { necessidades metabólicas. }\end{array}$ \\
\hline $\begin{array}{l}4 \text { - Atividade/ } \\
\text { repouso }\end{array}$ & Insônia (00095) & $\begin{array}{c}\text { Distúrbio na quantidade e qualidade do sono que prejudica o } \\
\text { desempenho normal das funções da vida diária. }\end{array}$ \\
\hline $\begin{array}{l}4-\text { Atividade/ } \\
\text { repouso }\end{array}$ & $\begin{array}{l}\text { Mobilidade física } \\
\text { prejudicada (00085) }\end{array}$ & $\begin{array}{l}\text { Limitação no movimento independente e voluntário do corpo } \\
\text { ou de uma ou mais extremidades. }\end{array}$ \\
\hline $\begin{array}{l}4 \text { - Atividade/ } \\
\text { repouso }\end{array}$ & Fadiga (00093) & $\begin{array}{l}\text { Sensação opressiva e prolongada de exaustão e capacidade } \\
\text { diminuída de realizar trabalho físico e mental no nível } \\
\text { habitual. }\end{array}$ \\
\hline $\begin{array}{l}4 \text { - Atividade/ } \\
\text { repouso }\end{array}$ & $\begin{array}{l}\text { Padrão respiratório } \\
\text { ineficaz (00032) }\end{array}$ & $\begin{array}{l}\text { Inspiração e/ou expiração que não proporciona ventilação } \\
\text { adequada. }\end{array}$ \\
\hline $\begin{array}{l}4 \text { - Atividade/ } \\
\text { repouso }\end{array}$ & $\begin{array}{l}\text { Intolerância à atividade } \\
(00092)\end{array}$ & $\begin{array}{l}\text { Energia fisiológica ou psicológica insuficiente para suportar } \\
\text { ou completar as atividades diárias requeridas ou desejadas. }\end{array}$ \\
\hline $\begin{array}{l}4 \text { - Atividade/ } \\
\text { repouso }\end{array}$ & $\begin{array}{l}\text { Ventilação espontânea } \\
\text { prejudicada (00033) }\end{array}$ & $\begin{array}{c}\text { Incapacidade de iniciar e/ou manter respiração independente } \\
\text { que seja adequada para sustentação da vida. }\end{array}$ \\
\hline $\begin{array}{l}11 \text { - Segurança/ } \\
\text { proteção }\end{array}$ & $\begin{array}{l}\text { Desobstrução ineficaz } \\
\text { das vias aéreas } \\
(00031)\end{array}$ & $\begin{array}{l}\text { Incapacidade de eliminar secreções ou obstruções do trato } \\
\text { respiratório para manter a via aérea desobstruída. }\end{array}$ \\
\hline $\begin{array}{l}11 \text { - Segurança/ } \\
\text { proteção }\end{array}$ & $\begin{array}{l}\text { Risco de integridade } \\
\text { tissular prejudicada } \\
\text { (00248) }\end{array}$ & $\begin{array}{c}\text { Suscetibilidade a dano em membrana mucosa, córnea, } \\
\text { sistema tegumentar, fáscia muscular, músculo, tendão, osso, } \\
\text { cartilagem, cápsula articular e/ou ligamento que pode } \\
\text { comprometer a saúde. }\end{array}$ \\
\hline $\begin{array}{l}11 \text { - Segurança/ } \\
\text { proteção }\end{array}$ & Hipertermia (00007) & $\begin{array}{c}\text { Temperatura corporal central acima dos parâmetros diurnos } \\
\text { normais devido a falha na termorregulação. }\end{array}$ \\
\hline 12 - Conforto & $\begin{array}{l}\text { Conforto prejudicado } \\
\quad(00214)\end{array}$ & $\begin{array}{l}\text { Percepção de falta de conforto, de alívio e de transcendência } \\
\text { nas dimensões física, psicoespiritual, ambiental, cultural e/ou } \\
\text { social. }\end{array}$ \\
\hline 12 - Conforto & Dor aguda (00132) & $\begin{array}{l}\text { Experiência sensorial e emocional desagradável associada a } \\
\text { lesão tissular real ou potencial, ou descrita em termos de tal } \\
\text { lesão (International Association for the Study of Pain); início } \\
\text { súbito ou lento, de intensidade leve a intensa, com término } \\
\text { antecipado ou previsível e com duração menor que } 3 \text { meses. }\end{array}$ \\
\hline
\end{tabular}

Fonte: Andrade TRSF, et al., 2020; dados extraídos de NANDA-I (2018).

O quadro a seguir foi organizado com os diagnósticos de enfermagem, manifestações clínicas que se enquadram nos diagnósticos, fatores relacionados/fatores de risco e características definidoras de acordo com os sinais e sintomas elencados. É importante destacar que alguns diagnósticos de enfermagem se enquadravam em mais de uma manifestação clínica (Tabela 2). 
Tabela 2 - Principais diagnósticos de enfermagem identificados de acordo com as manifestações clínicas da COVID-19. Aracaju-SE, 2020.

\begin{tabular}{|c|c|c|c|}
\hline $\begin{array}{c}\text { Diagnósticos de } \\
\text { enfermagem }\end{array}$ & $\begin{array}{l}\text { Manifestações } \\
\text { clínicas }\end{array}$ & $\begin{array}{c}\text { Fatores relacionados/ } \\
\text { Fatores de risco }\end{array}$ & Características definidoras \\
\hline \multirow{2}{*}{$\begin{array}{l}\text { Deglutição } \\
\text { prejudicada }\end{array}$} & Tosse & Inflamação & \multirow{2}{*}{ Tosse } \\
\hline & Dor de garganta & Dor & \\
\hline \multirow{4}{*}{$\begin{array}{l}\text { Nutrição } \\
\text { desequilibrada: } \\
\text { menor do que as } \\
\text { necessidades } \\
\text { corporais }\end{array}$} & & \multirow{4}{*}{$\begin{array}{l}\text { Ingestão alimentar } \\
\text { insuficiente } \\
\text { Dor na deglutição }\end{array}$} & \multirow{4}{*}{$\begin{array}{l}\text { Alteração no paladar } \\
\text { Ingestão de alimentos menor que } \\
\text { a ingestão diária recomendada }\end{array}$} \\
\hline & Tosse & & \\
\hline & & & \\
\hline & Dor de garganta & & \\
\hline \multirow{4}{*}{ Insônia } & & \multirow{4}{*}{$\begin{array}{c}\text { Desconforto físico } \\
\text { Ansiedade }\end{array}$} & \multirow{4}{*}{$\begin{array}{l}\text { Dificuldade para manter o sono } \\
\text { Alteração no padrão de sono } \\
\text { Energia insuficiente } \\
\text { Insatisfação com o sono }\end{array}$} \\
\hline & losse & & \\
\hline & & & \\
\hline & Dispneia & & \\
\hline \multirow{3}{*}{$\begin{array}{l}\text { Mobilidade física } \\
\text { prejudicada }\end{array}$} & & \multirow{3}{*}{$\begin{array}{c}\text { Dor } \\
\text { Intolerância à atividade }\end{array}$} & \multirow{3}{*}{$\begin{array}{c}\text { Desconforto } \\
\text { Dispneia ao esforço } \\
\text { Redução na amplitude de } \\
\text { movimentos }\end{array}$} \\
\hline & Mialgia & & \\
\hline & Fadiga & & \\
\hline \multirow{2}{*}{ Fadiga } & Fadiga & \multirow{2}{*}{$\begin{array}{l}\text { Estressores } \\
\text { Ansiedade }\end{array}$} & Cansaço \\
\hline & Mialgia & & $\begin{array}{l}\text { Letargia } \\
\text { Energia insuficiente }\end{array}$ \\
\hline $\begin{array}{c}\text { Padrão respiratório } \\
\text { ineficaz }\end{array}$ & Dispneia & $\begin{array}{c}\text { Fadiga da musculatura } \\
\text { respiratória } \\
\text { Dor }\end{array}$ & $\begin{array}{c}\text { Dispneia } \\
\text { Padrão respiratório anormal } \\
\text { Uso de musculatura acessória }\end{array}$ \\
\hline \multirow{2}{*}{$\begin{array}{l}\text { Intolerância à } \\
\text { atividade }\end{array}$} & Mialgia & \multirow{2}{*}{ Mal-estar geral } & Dispneia ao esforço \\
\hline & Fadiga & & Desconforto ao esforço \\
\hline $\begin{array}{l}\text { Ventilação } \\
\text { espontânea } \\
\text { prejudicada }\end{array}$ & Dispneia & $\begin{array}{l}\text { Fadiga da musculatura } \\
\text { respiratória }\end{array}$ & $\begin{array}{c}\text { Apreensão } \\
\text { Aumento da frequência cardíaca } \\
\text { Uso aumentado da musculatura } \\
\text { acessória } \\
\text { Dispneia } \\
\text { Inquietação }\end{array}$ \\
\hline \multirow{2}{*}{$\begin{array}{c}\text { Desobstrução } \\
\text { ineficaz das vias } \\
\text { aéreas }\end{array}$} & Dispneia & \multirow{2}{*}{$\begin{array}{l}\text { Secreções retidas } \\
\text { Muco excessivo }\end{array}$} & Inquietação \\
\hline & Tosse & & $\begin{array}{l}\text { Tosse ineficaz } \\
\text { Dispneia }\end{array}$ \\
\hline $\begin{array}{l}\text { Risco de integridade } \\
\text { tissular prejudicada }\end{array}$ & Coriza & $\begin{array}{c}\text { Movimentos repetitivos } \\
\text { de higiene na região } \\
\text { nasal }\end{array}$ & $T_{1}$ \\
\hline Hipertermia & Febre & Doença & Pele quente ao toque \\
\hline \multirow{5}{*}{ Conforto prejudicado } & Mialgia & \multirow{5}{*}{$\begin{array}{c}\text { Manifestações clínicas } \\
\text { da doença }\end{array}$} & Ansiedade \\
\hline & Coriza & & Desconforto \\
\hline & Dispneia & & Alteração no padrão de sono \\
\hline & Fadiga & & Irritabilidade \\
\hline & Tosse & & Inquietação \\
\hline \multirow{3}{*}{ Dor aguda } & Mialgia & \multirow{3}{*}{ Agente biológico lesivo } & Alteração no apetite \\
\hline & Dor de garganta & & Alteração no parâmetro fisiológico \\
\hline & Tosse & & Expressão facial de dor \\
\hline
\end{tabular}

Fonte: Andrade TRSF, et al., 2020; dados extraídos de Brasil (2020a) e NANDA-I (2018). 


\section{DISCUSSÃO}

A infecção pelo coronavírus pode vir acompanhada de diversas manifestações clínicas ou até mesmo de forma assintomática. As informações mais recentes encontradas na literatura sobre a COVID-19 descrevem que cerca de $80 \%$ da população apresenta a doença de forma leve, enquanto os casos graves atingem principalmente indivíduos que possuem comorbidades como obesidade, diabetes mellitus, doenças cardiovasculares, neoplasias e doenças respiratórias crônicas. Dessa forma, diante da diversidade de apresentações clínicas que podem ser encontradas nos indivíduos infectados pelo coronavírus, é importante compreender os sinais e sintomas que envolvem a doença e a forma que impactam o organismo (BRASIL, 2020b).

O atendimento inicial ao paciente infectado pelo coronavírus deve incluir a avaliação das manifestações clínicas, bem como o histórico recente do paciente. Além disso, deve ser realizado um exame físico de qualidade, com todas as medidas de segurança para evitar a contaminação do profissional, e solicitação de exames laboratoriais e de imagem de acordo com a necessidade de cada caso clínico para definição do diagnóstico. É importante salientar que todos os procedimentos realizados nos pacientes devem seguir protocolos rígidos de segurança para evitar contaminação do local e dos demais envolvidos no processo de cuidado (BRASIL, 2020a).

A tosse representa uma das queixas mais comuns dos pacientes com suspeita ou confirmação da COVID19 nos serviços da saúde, conforme identificado no estudo de Huang C, et al. (2020), onde 76\% dos pacientes apresentavam tosse, bem como no de Chen T, et al. (2020) com 66\% a 70\% e no de Mao L, et al. (2020) com $50 \%$. Para a alteração clínica tosse foram elencados os diagnósticos de enfermagem "deglutição prejudicada", "nutrição desequilibrada: menor do que as necessidades corporais", "desobstrução ineficaz das vias aéreas", "insônia", "conforto prejudicado" e "dor aguda", visto que todos esses poderão estar presentes devido ao caráter irritativo da tosse, que causa desconforto e interfere no bem-estar e nas necessidades fisiológicas quando acontece de forma exacerbada.

Dessa forma, é importante observar os desconfortos trazidos pela tosse para o paciente, principalmente quando afeta em algum aspecto do tratamento clínico, buscando constantemente melhorias do estado de saúde e recuperação eficaz.

A febre, também amplamente presente nos relatos dos pacientes infectados pelo coronavírus, foi identificada em 98\% dos pacientes na pesquisa de Huang C, et al. (2020), 72,8\% no de Giacomelli A, et al. (2020) e 61,7\% no de Mao L, et al. (2020). O diagnóstico de enfermagem escolhido foi "hipertermia", visto que representa a elevação da temperatura corporal. A febre se comporta como um sinal de alerta no organismo, indicando que há algum processo inflamatório acontecendo e que o sistema de defesa precisa ser ativado (GOMES BRB, 2017).

Por isso, é importante monitorar periodicamente a temperatura do paciente e realizar as intervenções necessárias diante da sua elevação. Cabe ainda destacar a importância de observar as características da febre, como sua duração, periodicidade e intensidade, com o intuito de identificar o perfil de apresentação da manifestação clínica no paciente, bem como os meios de controle mais eficazes.

Sobre a mialgia, a literatura destaca uma epidemiologia que varia de $30 \%$ no estudo de Wang $Z$, et al. (2020), 28,5\% na metanálise desenvolvida por Terceiro D e Vietto V (2020) e 14,9\% no trabalho de Guan W, et al. (2020). Os diagnósticos de enfermagem selecionados foram "mobilidade física prejudicada", "fadiga", "intolerância à atividade", "conforto prejudicado" e "dor aguda", visto que a dor muscular generalizada ou localizada causada pela doença pode levar a um mal-estar geral e indisposição que interfere em diversas atividades do indivíduo.

Nesse contexto, é importante caracterizar o quadro de mialgia relatado pelo paciente, buscando identificar duração, localização e intensidade, de modo que sejam implementadas as melhores intervenções para promover conforto e bem-estar. 
A dispneia, quadro clínico que exige avaliação detalhada devido ao risco de agravamento respiratório, foi identificada em $55 \%$ dos pacientes no trabalho de Huang C, et al. (2020), em 25,4\% no de Giacomelli A, et al. (2020) e em 21,9\% no de Li L, et al. (2020). "Insônia", "padrão respiratório ineficaz", "ventilação espontânea prejudicada", "desobstrução ineficaz das vias aéreas" e "conforto prejudicado" foram os diagnósticos de enfermagem identificados. Tanto questões fisiológicas relacionadas ao sistema respiratório quanto atividades de repouso e conforto são prejudicadas por essa manifestação clínica, uma vez que gera desconforto respiratório, ansiedade e medo, o que interfere fortemente no estado de saúde do paciente. Com isso, é essencial que sejam realizadas orientações adequadas sobre a necessidade de cuidados especializados nos casos em que o indivíduo tenha suas atividades de vida diárias prejudicadas pela dispneia.

A dor de garganta se apresenta em menor escala nas queixas dos pacientes infectados pelo coronavírus. Jin X, et al. (2020) identificaram essa manifestação clínica em 15,2\% dos indivíduos, Guan W, et al. (2020) em 13,9\% e Bhatraju PK, et al. (2020) em $8 \%$. Três diagnósticos de enfermagem foram selecionados para essa manifestação, sendo eles "deglutição prejudicada", "nutrição desequilibrada: menor do que as necessidades corporais" e "dor aguda". O desconforto presente na garganta interfere na deglutição e consequentemente na ingestão de alimentos, podendo levar a perda do apetite devido à dificuldade e dor. Nessa perspectiva, estar atento as queixas do paciente e observar seu padrão de ingestão alimentar é uma ação importante, principalmente pela relevância que a nutrição de qualidade possui na resposta de recuperação do indivíduo. Além disso, avaliar o nível da dor é importante para implementação de medidas de alívio conforme necessidade.

A fadiga é encontrada em valores mais expressivos como quadro clínico do paciente com COVID-19, sendo visto em $42 \%$ dos avaliados no trabalho de Wang Z, et al. (2020), 38\% no de Terceiro D e Vietto V (2020) e 31,1\% no de Jin X, et al. (2020). Os diagnósticos de enfermagem eleitos foram "mobilidade física prejudicada", "fadiga", "intolerância à atividade" e "conforto prejudicado". A fadiga gera uma situação de cansaço excessivo e falta de energia que interfere na realização das atividades e prejudica o desenvolvimento de ações essenciais para o organismo. Assim como na mialgia, é importante avaliar até que ponto a fadiga interfere na vida do indivíduo e buscar promover conforto para que a recuperação ocorra de maneira mais eficaz.

A coriza, identificada como uma manifestação clínica menos comum, foi encontrada em $35 \%$ dos pacientes no trabalho de Speth MM, et al. (2020), em 17\% no de Bhatraju PK, et al. (2020) e em 4\% no estudo de Chen $\mathrm{N}$, et al. (2020). Os diagnósticos de enfermagem escolhidos foram "risco de integridade tissular prejudicada" e "conforto prejudicado". A coriza gera uma sensação de desconforto pela congestão nasal e necessidade constante de higiene da região nasal, o que pode levar a lesão da pele. Sendo assim, é necessário que sejam dadas orientações sobre a maneira correta de manipular as secreções nasais, bem como métodos de higiene que não lesionem a mucosa, de modo a evitar ferimentos que podem servir como portas de entrada para outras infecções.

Além das manifestações clínicas abordadas no estudo, existem outras queixas que podem ser apresentadas pelos pacientes no momento da consulta de triagem, durante a progressão da doença e até mesmo no internamento. Assim, cabe ao profissional atuante na linha de frente realizar os treinamentos técnicos e teóricos que vêm sendo oferecidos pelo Ministério da Saúde e por outras instituições para que haja um aperfeiçoamento constante, viabilizando boas práticas de saúde que confiram proteção aos profissionais da equipe e aos pacientes. Do mesmo modo, as instituições devem se mostrar atuantes na proteção e capacitação da sua equipe, disponibilizando também meios para realização das atividades adequadas e garantindo os melhores resultados para todos os atores envolvidos na luta contra o coronavírus (BRASIL, 2020a; BRASIL, 2020b).

Nessa perspectiva, para que seja possível a implementação da SAE e do PE, é necessário que os profissionais de enfermagem disponham de um suporte e apoio por parte da gestão de saúde, tanto através de treinamentos como de recursos humanos e materiais para o desempenho das atividades. Assim será possível elaborar diagnósticos de enfermagem precisos para os pacientes infectados pelo coronavírus e traçar o planejamento e as intervenções adequadas para o paciente de forma individual, garantindo um cuidado humanizado e com melhores resultados. 


\section{CONSIDERAÇÕES FINAIS}

A COVID-19 surgiu de forma repentina na sociedade, se espalhou por todo o mundo e atinge um número alarmante de infectados, além de causar milhares de óbitos. Dessa forma, é importante destacar a relevância de uma assistência adequada aos pacientes, dado que a boa sistematização do cuidado influencia diretamente no processo de melhoria do quadro clínico. Diante do exposto, a SAE e o PE se destacam por permitir o cuidado holístico e individualizado ao paciente com COVID-19, com acompanhamento diário e estruturado. Os diagnósticos de enfermagem elencados neste estudo para as manifestações clínicas mais comuns da doença englobaram fatores nutricionais, respiratórios, de conforto e de bem estar, todos esses elementos importantes para a recuperação do indivíduo infectado pelo coronavírus.

\section{REFERÊNCIAS}

1. BARROS ALBL, et al. Processo de Enfermagem: guia para a prática. Conselho Regional de Enfermagem de São Paulo. São Paulo: COREN-SP, 2015; 113p.

2. BHATRAJU PK, et al. Covid-19 in critically III patients in the Seattle Region - case series. The New England Journal of Medicine, 2020; 382: 2012-2022.

3. BITENCOURT GR, et al. Padrões funcionais de saúde em adultos com covid-19 na terapia intensiva: Fundamentando diagnósticos de enfermagem. Internacional Journal of Development Research, 2020; 10(6): 36540-36544.

4. BRASIL. Ministério da Saúde. Diretrizes para Diagnóstico e Tratamento da COVID-19. Brasília: Ministério da Saúde; 2020 a.

5. BRASIL. Ministério da Saúde. Protocolo de Manejo Clínico do Coronavírus (Covid-19) na Atenção Primária à Saúde. Brasília: Ministério da Saúde; 2020b.

6. CHEN N, et al. Epidemiological and clinical characteristics of 99 cases of 2019 novel coronavirus pneumonia in Wuhan, China: a descriptive study. The Lancet, 2020; 395(10223): 507-513.

7. CHEN T, et al. Clinical characteristics of 113 deceased patients with coronavirus disease 2019: retrospective study. BMJ, 2020; 368: m1091.

8. DANTAS TP, et al. Diagnósticos de enfermagem para pacientes com COVID-19. Journal Health NPEPS, 2020; 5(1): 396-416.

9. FIOCRUZ. 2020. Fundação Oswaldo Cruz. Plano de Contingência da Fiocruz diante da pandemia da doença pelo SARS-Cov-2 (Covid-19). Rio de Janeiro: FIOCRUZ, 2020; 79p.

10. GIACOMELLI A, et al. Self-reported olfactory and taste disorders in patients with severe acute respiratory coronavirus 2 infection: a cross-sectional study. Clinical Infectious Diseases, 2020; 71(15): 889-890.

11. GOMES BRB. Análise da produção de Espécies reativas de Oxigênio e Nitrogênio durante a febre e a antipirese em ratos. Dissertação (Mestrado em Patologia Molecular) - Programa de Pós-Graduação em Patologia Molecular. Faculdade de Medicina da Universidade de Brasília, Brasília, 2017; 75p.

12. GUAN W, et al. Clinical characteristics of coronavirus disease 2019 in China. The New England Journal of Medicine, 2020; 382(18): 1708-1720.

13. HUANG C, et al. Clinical features of patients infected with 2019 novel coronavirus in Wuhan, China. Lancet, 2020; 395(10223): 497-506.

14. JIN X, et al. Epidemiological, clinical and virological characteristics of 74 cases of coronavirus-infected disease 2019 (COVID-19) with gastrointestinal symptoms. BMJ, 2020; 69(6): 1002-1009.

15. LI L, et al. COVID-19 patients' clinical characteristics, discharge rate, and fatality rate of meta-analysis. Journal of Medical Virology. 2020; 92(6): 577-583.

16. MAO L, et al. Neurologic manifestations of hospitalized patients with coronavirus disease 2019 in Wuhan, China. JAMA Neurology, 2020; 77(6): 683-690.

17. MONTE LM, et al. Complicações atípicas e características clínico-epidemiológicas do COVID-19: uma revisão integrativa. Revista Eletrônica Acervo em Saúde, 2020; 46: e3699.

18. NANDA-I. Diagnósticos de Enfermagem da NANDA: Definições e Classificações. 2018-2020. 11ª ed. Porto Alegre: Artmed, 2018; 488p.

19. OPAS. 2020. Organização Pan-Americana da Saúde. Folha informativa - COVID-19 (doença causada pelo novo coronavírus). https://www.paho.org/bra/index.php?option=com_content\&view=article\&id=6101:covid19\&Itemid=875 Acesso em: 22 jul. 2020.

20. SPETH MM, et al. Olfactory dysfunction and sinonasal symptomatology in covid-19: prevalence, severity, timing, and associated characteristics. Otolaryngology - Head and Neck Surgery, 2020; 163(1): 114-120.

21. TANNURE MC, PINHEIRO AM. SAE - Sistematização da Assistência de Enfermagem: guia prático. 2ª̣ ed. Rio de Janeiro: Guanabara Koogan, 2010; 312p.

22. TERCEIRO DR, VIETTO V. COVID-19: presentación clínica en adultos. Evidencia - Actualización en la Práctica Ambulatoria, 2020; 23(2): e002042.

23. WANG Z, et al. Clinical features of 69 cases with coronavirus disease 2019 in Wuhan, China. Clinical Infectious Diseases, 2020; 71(15): 769-777.

24. YOSHIKAWA G, CASTRO C. Manual de semiologia médica: a prática do exame físico. Belém: EDUEPA, 2015. 473p. 